\title{
The 3-dimensional anatomy of the North-Western Marsupial Mole (Notoryctes caurinus Thomas 1920) using computed tomography, $X$-ray and magnetic resonance imaging
}

\author{
Natalie Warburton ${ }^{1}$, Christopher Wood ${ }^{2}$, Christopher Lloyd ${ }^{2}$, Swithin Song ${ }^{2}$ and Philip Withers ${ }^{1}$ \\ ${ }^{1}$ Department of Zoology, University of Western Australia \\ Crawley, Western Australia, 6009. E-mail: nwarbo@cyllene.uwa.edu.au \\ ${ }^{2}$ Department of Radiology, Royal Perth Hospital \\ Wellington Street, Perth, Western Australia, 6000
}

\begin{abstract}
The 3-D skeletal images obtained from reconstruction of CT scans and X-rays, and soft-tissue images produced by MRI, provide invaluable information of the internal and gross anatomy of the north-western marsupial mole (Notoryctes caurinus).

The conical skull, which is quite thin-walled dorsally and anteriorly but strong in the basicranial region, has little evidence of the orbit or zygomatic arch, and the smoothly-curved posterior region has no sagittal or occipital crests. The vertebral column is remarkably strengthened, and in lateral view has an unusual flat-shape. The cervical vertebrae appear to be greatly compressed; 4 or 5 are completely fused (which is unique among marsupials). The thoracic vertebrae are fairly robust with large neural spines. The lumbar vertebrae are distinct, becoming large posteriorly towards the pelvis. The sacral vertebrae are greatly expanded in size and are fused with the pelvis. Particularly in the middle of the tail, the caudal vertebrae are greatly developed, with large transverse processes and chevron bones. The pectoral girdle is very anterior, with the shoulder articulation level with the anterior cervical vertebrae just behind the skull, and low on the side of the body. The humerus is robust, and the radius and ulna are very short. The bones of the pelvis are highly derived, and fused to sacral vertebrae. The epipubic bones are small and not ossified. An ossified patella is present and it has an unusual large triangular keel.

The most apparent soft-tissue structure by MRI is a large amount of subcutaneous fat, particularly around the ventral surface of the pelvis but also dorsal to the pelvis and anteriorly around the shoulders. The major muscle groups are visible, but distinction between individual muscles is not possible except for the very large muscles of the thigh, upper arm and base of the tail. The muscles of the tail are strongly developed, more so ventrally than dorsally.
\end{abstract}

\section{INTRODUCTION}

Computed tomography (CT scan) and magnetic resonance imaging (MRI) are used extensively in medical diagnostics and research and the techniques have also been incorporated into the veterinary discipline in clinical situations. However, the zoological use of such technology in more basic anatomical research has been rather limited. The few anatomical investigations incorporating CT and MRI technology include descriptions of the internal anatomy of the feline abdomen (Samii et al. 1999), and the brain and coelomic cavity of the domestic pigeon (Romagnano et al. 1996). The techniques have also been used in paleaontological studies, for example the measurements of brain size in Allosaurus (Rogers 1999). This research uses CT scan and MRI techniques to investigate the anatomy of the marsupial mole.

The anatomy of the North-western marsupial mole, Notoryctes caurinus (Marsupialia: Notoryctidae), has not been studied in any detail since its original description by Thomas (1920). In fact the only published research for this remarkable fossorial marsupial since then is the physiological study of Withers et al. (2000) and a natural history account by Thompson et al. (2000-2001). Very few preserved specimens of $N$. caurinus are available, so it is important to glean as much anatomical information as non-destructively as possible from the few specimens that are available. The purpose of this paper is to provide an account of the usefulness of CT scan, X-ray, and MRI to describe $N$. caurinus, a small (mass $\sim 30-35 \mathrm{~g}$ ) and highly specialized marsupial. 


\section{METHODS}

The primary specimen of $N$. caurinus which we examined was from the Western Australian Museum (specimen number M 44175). Capture locality was Cotton Creek (Newman-Canning Stock Route road; $4 / 3 / 1999 ; 22^{\circ} 59^{\prime} 122^{\circ} 23^{\prime}$ ). The specimen was formalin fixed and preserved in $70 \%$ ethanol. The specimen was male, with a total body length of $10.5 \mathrm{~cm}$ and mass of approximately $35 \mathrm{~g}$.

$\mathrm{CT}$ and MRI scans were performed at Royal Perth Hospital, Perth, Western Australia. The CT scans were made with on a GE Cti scanner (General Electric Medical Systems, Milwaukee, USA) using a helical technique with a slice collimation of $1 \mathrm{~mm}$, and a pitch of 1:1. Images were reconstructed at an interval of $0.3 \mathrm{~mm}$ using soft tissue and bone algorithms. 3-D volume rendered images were also obtained using the AW 3.0 workstation.

MRI scans were performed using a Marconi 1.0T MR scanner (Marconi Medical Systems, Highland Heights, Ohio, USA) using a 3-D RF FAST sequence with $1.4 \mathrm{~mm}$ slices, flip angle of 35 degrees, TR of $24 \mathrm{~ms}$, TE of $6 \mathrm{~ms}$, field of view of $12 \mathrm{~cm}, 192 \times 256$ matrix, one signal average. Images were acquired in the coronal, transverse and sagittal planes. The specimen was placed in a dedicated (human) knee coil.

X-rays were taken by Dr Ken Aplin of the Western Australian Museum, using standard x-ray film and processing techniques. The fine details of the images were verified by dissection.

\section{RESULTS AND DISCUSSION}

Computed tomography was most useful for examining hard, bony structures of the body (Figure 1 ). Such 3-D images of the skeleton of $N$. caurinus are useful because no fully-articulated specimen of this species is locally available, and because the functional form of its skeleton cannot necessarily be inferred from simple reconstruction of disarticulated bones because of its unique fossorial lifestyle among marsupials. Thus, 3-D computer reconstructions of $\mathrm{CT}$ scans provide invaluable information concerning the normal in situ skeletal arrangement. $X$-rays were necessary to provide fine detail of skeletal structures. MRI was useful in viewing the internal arrangement of soft tissue structures.

\section{3-D Skeletal Reconstruction}

The axial skeleton, comprising the skull, vertebral column, sternum and ribs, and tail of the northwestern marsupial mole is highly adapted for its fossorial lifestyle (Figure 1). From the lateral view of the skeletal image, the skull is conical in shape (Figure 1C). The skull of N. caurinus appears to be quite thin-walled dorsally and anteriorly, but strong in the basicranial region. Teeth are not apparent on the CT scans because they are too small to be resolved by a scan of $1.0 \mathrm{~mm}$ slice size. The skull, including the major contours and teeth, is finely imaged by $\mathrm{X}$-ray (Figure 2). The rostrum curves ventrally slightly, anterior to the molar teeth. There is little evidence of the orbit or zygomatic arch. The posterior region of the skull is smoothly curved with no sagittal or occipital crests.

The vertebral column is remarkably strengthened, and in lateral view has an unusually flat shape (Figure 1C). Overall, the form of the vertebral column appears highly specialised for burrowing. While CT resolution is not quite adequate for the fine detail of vertebrae, it is apparent that they are very robust. The cervical vertebrae are obscured by the pectoral girdle, but appear to be greatly compressed and follow a deeply concave path to the base of the skull. This unusual form of the cervical vertebrae is best seen from the lateral view of the skeletal images (Figures 1C, 2B). Fusion of the cervical vertebrae is extremely unusual for mammals, and is unique among marsupials for the marsupial mole. The thoracic vertebrae are not seen as separate entities in the 3-D skeletal images. The shape of the vertebral column in the thoracic region follows a sharp incline behind the pectoral girdle and then flattens out considerably. The vertebral column of more generalised quadrupeds follows a wider curve than that apparent for $N$. caurinus (Slijper 1946).

The lumbar region is best seen from dorsal view (Figures 1A, 2A). The lumbar vertebrae are distinct, becoming large posteriorly towards the pelvis. Their transverse processes can just be discerned on $\mathrm{CT}$ images. Like the posterior thoracic vertebrae, the lumbar vertebral region is very flat in shape. The sacral vertebrae are greatly expanded in size and are fused with the pelvis. The wide metapophyses are broadened to form a shield-shaped arrangement when viewed from the dorsal aspect (Stirling, 1891; Chapman, 1919).

An unusual feature of the vertebral column from the dorsal view is the arrangement of vertebrae in the tail (Figure 1). Particularly in the middle region of the tail, these caudal vertebrae are greatly developed; large transverse processes and chevron bones (apparently fused to the vertebral bodies) are evident on the CT scans. From the lateral aspect, the caudal vertebrae are almost as large as the thoracic and lumbar vertebrae, which is most unusual. Generally, caudal vertebrae of mammals are smaller and simpler in form than the thoracic or lumbar vertebrae. The large size of the marsupial mole's caudal vertebrae, the development of transverse processes for muscle attachment, and the large size of the chevron bones, suggest that the tail is highly mobile, muscular and probably involved in burrowing locomotion (see Withers et al., 2000). 


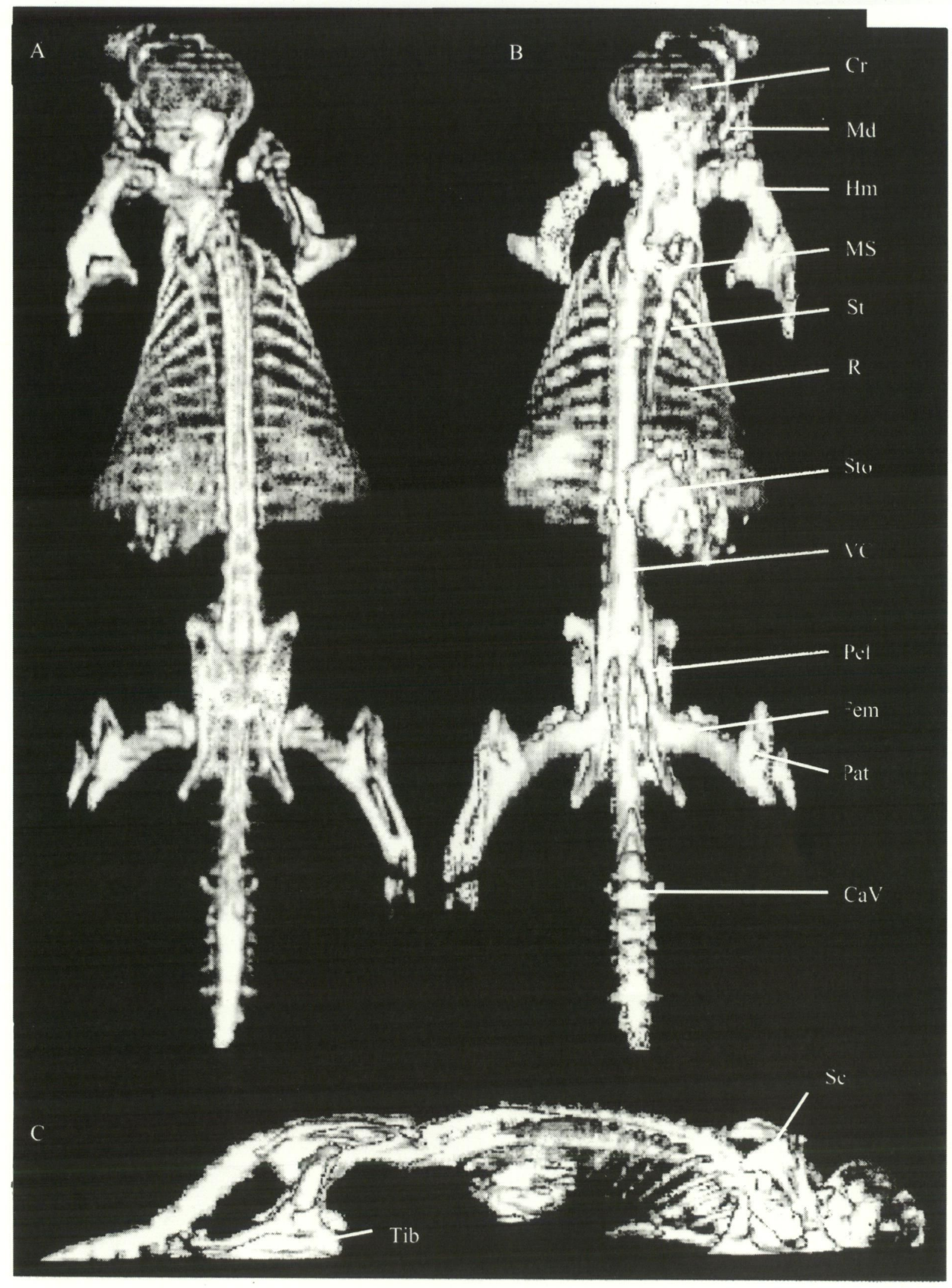

Figure 1 Computed tomography images of the three-dimensional anatomy of the North-Western Marsupial Mole, Notoryctes caurinus; a dorsal, b ventral, and c lateral views. Caudal vertebrae (CaV), cranium (Cr), femur $(\mathrm{Fem})$, humerus $(\mathrm{Hm})$, mandible (Ma), manubrium sterni (MS), patella (Pat), pelvis (Pel), ribs (r), scapula $(\mathrm{Sc})$, sternum (St), stomach (St), tibia (Tib), vertebral column (VC). 


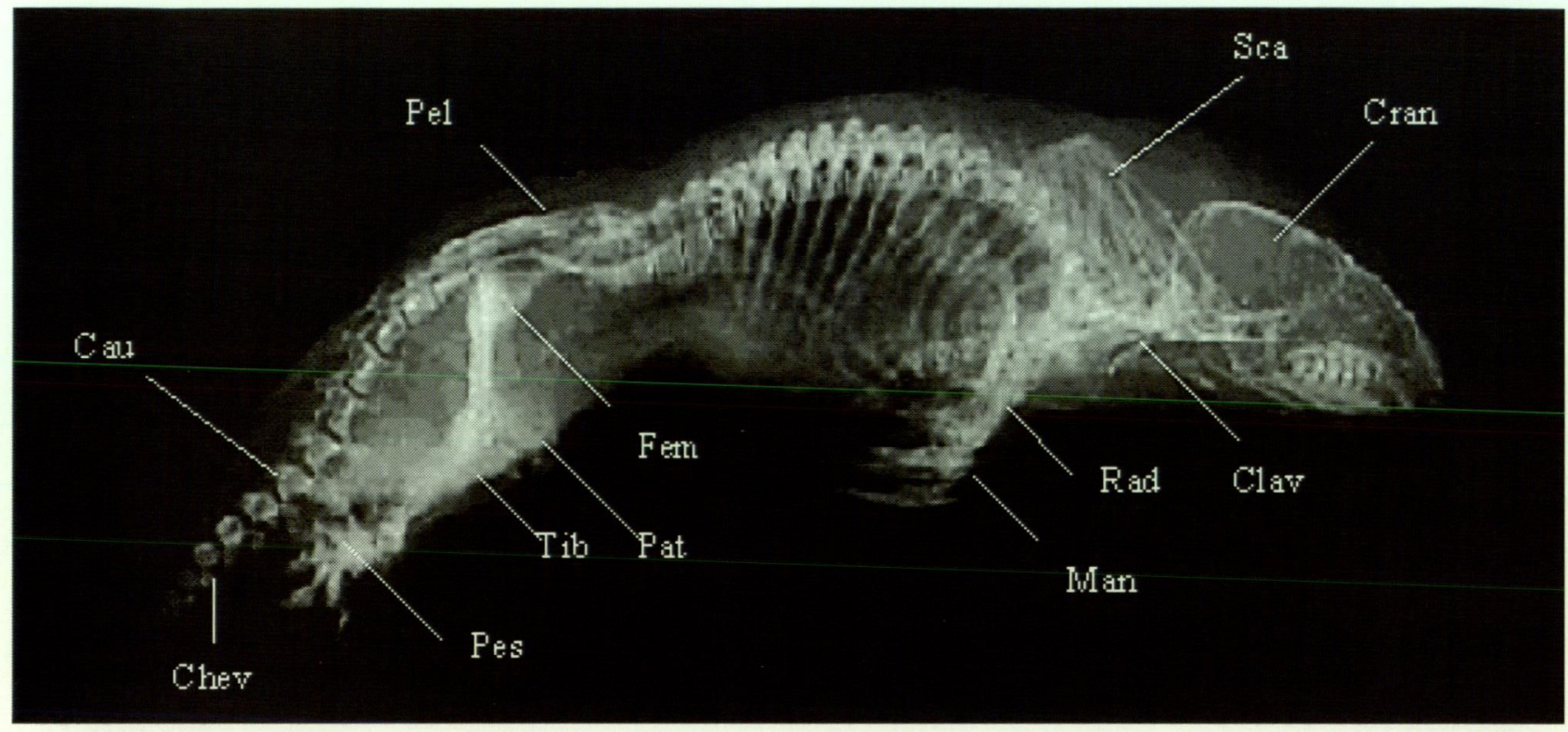

Figure 2 X-ray of the North-Western Marsupial Mole, Notoryctes caurinus (WAM M41482); lateral view. Actual body length (snout-tail) $=102 \mathrm{~mm}$. Legend: caudal vertebrae (Cau), chevron bones (Chev), clavicle (Clav), cranium (Cran), femur (Fem), manus (Man), patella (Pat), pelvis (Pel), pes (Pes), radius (Rad), scapula (Sca), tibia (Tib).

The ribs and sternum can be clearly seen in the CT skeletal images. The rib cage is in the form of a wide cone, being very narrow anteriorly and expanding quite widely posteriorly (Figure 1A, B). The ribs appear to become less dense posteriorly. The costal cartilages, which join the ribs to the sternum, cannot be seen. The anterior part of the sternum is characterised by two large lateral processes of the manubrium.

The appendicular skeleton includes the pectoral and pelvic limbs and their girdles. The highly modified morphology of these skeletal components in the marsupial mole can be seen in the 3-D skeletal reconstruction (Figure 1). Most noticeably, the pectoral girdle is quite anteriorly placed. The position of the shoulder articulation, of the glenoid region of the scapula and the humerus, appears level with the anterior cervical vertebrae, just behind the skull, rather than the more common position of level with the anterior thoracic vertebrae (Hildebrand, 1988). In the lateral view, it is apparent that the shoulder articulation is placed quite low on the side of the body. This anteroventral position of the pectoral girdle is characteristic of the position for other small fossorial mammals (Gambaryan and KeilanJaworowska, 1997).

The individual components of the pectoral girdle can be seen with reasonable clarity (Figure 1). It is apparent that the scapula is substantially modified, being narrow for the most part and fanning out posteriorly. The orientation of the scapula is also modified from the usual dorsal arrangement of the scapula in mammals (Hildebrand, 1988), occupying a more laterally-facing position (Figure 1C). The robust humerus can be seen most clearly in the dorsal views of the skeletal image; however this may include parts of the ulna included with the distal end of the humerus. Unfortunately the antebrachium (lower arm) and the manus were not represented in the skeletal images. This is quite unexpected as the bones of this region are relatively more robust than, for instance, the ribs, which were seen clearly in the skeletal reconstruction images.

The pelvic girdle is perhaps the most clear component of the skeleton. The bones of the pelvis are obviously highly derived and are fused to a number of the sacral vertebrae. The typically (for mammals) elongate innominate bones are broad and fused with expanded elements of the sacral vertebrae (Hildebrand, 1988). The fusion between the pelvis and the sacral vertebrae of marsupial moles is more extreme than in other placental burrowers, such as armadillos and pocket gophers (Chapman, 1919). It is evident that the pubic symphysis is either unfused or formed by very thin bone. The epipubic bones of marsupial moles are vestigial and are not seen in the CT scan images.

The hind limbs of $N$. caurinus extend almost laterally from the body, rather than ventrally, and the tibiae lie flat against the ground. Mammals generally have a parasaggital arrangement of the limbs under the body (Hildebrand, 1988); the abducted posture of the hindlimbs in marsupial moles is reminiscent of a more reptilian stance. The long bones of the limb are relatively robust, but no fusion between the elements has taken place. An unusual feature is the large triangular keel of the 

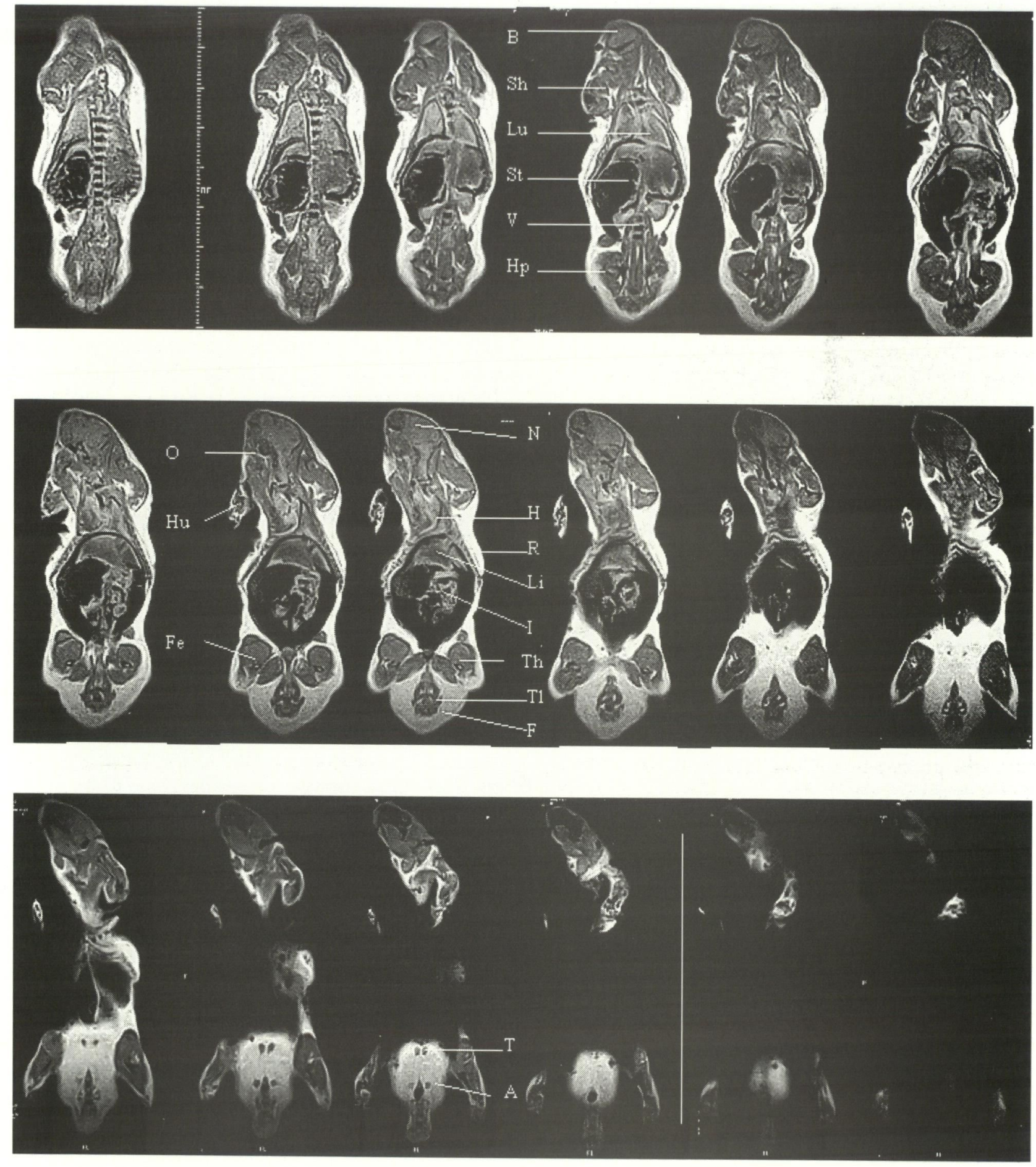

Figure 3 Magnetic resonance image of the three-dimensional anatomy of the North-Western Marsupial Mole, Notoryctes caurinus; sections through coronal view, dorsal to ventral. Organs visible: anal gland (A), brain (B), fat $(\mathrm{F})$, femur $(\mathrm{Fe})$ heart $(\mathrm{H})$, hip musculature $(\mathrm{Hp})$, humerus $(\mathrm{Hu})$, intestine (I) liver (Li), lungs (Lu), nose shield $(\mathrm{N})$, oral cavity $(\mathrm{O})$, rib cage $(\mathrm{R})$, shoulder musculature $(\mathrm{Sh})$, stomach $(\mathrm{St})$, tail musculature $(\mathrm{Ta})$, testes $(\mathrm{T})$, thigh musculature (Th), and vertebral column (V).

patella at the knee joint of the hind limbs (Figure 1C). Few other marsupials (only bandicoots) have an ossified patella, and none have one resembling the relatively large, irregular patella of Notoryctes. The tibia and fibula are pictured for the right leg only, but are clearly quite robust for an mammal of this size. Unfortunately, like the forelimb, the distal parts of the hind limb are not pictured in the 3-D skeletal image. Again this is unexpected as they have relatively robust bones. The unusual morphology of the hind limb, with the large triangular patella and expanded tibia is more clearly visible in the X-ray than the CT scan images. Like the carpus of the forelimb, the small bones of 
the pes are highly modified and difficult to identify even from $X$-ray images. However, it is interesting to note that the clawed digits of the foot are splayed, and held in a somewhat abducted and medially rotated posture, presumably to achieve the maximum surface area of the sole of the foot when burrowing.

\section{Soft Tissue Structure}

From all MRI views (Figure 3), the most apparent soft-structure is a large amount of subcutaneous fat on this specimen, particularly around the ventral surface of the pelvis but also dorsal to the pelvis and anteriorly around the shoulders. This very large amount of fat appears as a high signal intensity on the magnetic resonance images. Upon dissection, there was approximately four grams of subcutaneous fat - more than $10 \%$ of a 35 gram animal.

The largest organs of the digestive system, the liver, stomach and intestine, are easily distinguished. The abdominal cavity appears as very low signal intensity, oval-shaped structure in the mid-region of the animal, due to this space being air-filled as a result of an incision made in the skin for the penetration of formalin/alcohol for fixation of the internal organs. The liver is the most anterior organ of the abdomen, and multi-lobed in structure. The stomach appears quite dark on the left side of the abdominal cavity in the coronal sections. Also seen from CT scans, the stomach contains a large amount of sand resulting in the low signal intensity in MRI. The intestine fills the rest of the abdominal cavity and has a higher signal intensity than the stomach and liver. The smaller organs of the abdominal cavity, spleen, gall bladder, etc are not distinguishable. The oral cavity is also visible in the relevant coronal sections (Figure 3 ).

In the thoracic cavity, the heart is the only portion of the circulatory system that is clearly apparent in MRI. At particular levels of contrast it is possible to discern the chambers of the heart. Of the respiratory system the lungs are visible. Their triangular shape is best observed from the coronal sections (Figure 3).

The urinogenital system is poorly represented in the MRI due to the small size of these organs; only the testes are clear (in all views) and the kidneys in sagittal view. Posteriorly, lying in the mass of fat under the pelvis, the testes are seen very clearly, with good contrast between gonadal tissue and fat. It is difficult to assess the sex of marsupial moles externally as males have abdominal testes, so MRI provides a non-intrusive method for confirming that this specimen is male.

The brain is the only obvious component of the nervous system in the MRI images (Figure 3 ). With the MRI having a slice size of $1.4 \mathrm{~mm}$, it is possible that the spinal cord was missed altogether in coronal and sagittal sections.
The hard, bony structures of the skeletal system appear dark on the MRI. Clearly visible are sections through the skull, vertebral column, ribs, humeri and femora. The large size of the vertebrae is obvious and it is easier to distinguish adjacent vertebrae in MRI than CT scans. Surrounding the long bones of the limbs, the major muscle groups are visible, but distinction between individual muscles is not possible except for the very large muscles of the thigh, upper arm and base of the tail. In these areas the muscle bulk is enormous, much greater than for non-fossorial mammals of comparable size (personal observations). The extensive muscles attaching to the tail are quite unusual and would seem to be related to the strengthening of the caudal vertebrae as noted from the CT skeletal images. The muscles of the tail also appear to be more strongly developed ventrally (for flexion) than dorsally (for extension).

In summary, traditional $X$-rays provide detail of skeletal elements but are sometimes difficult to interpret because a three dimensional structure has been projected onto a two dimensional image. Reconstruction of $\mathrm{CT}$ scans results in a three dimensional image that reduces the problem of overlap. Also, with the CT skeletal reconstruction, it is possible to rotate the image to obtain the desired view, an impossible manipulation with $X$-ray images. MRI provides information of the anatomy of soft tissue structures by recording the differential contrast of the various soft tissues. Due to the small size of the marsupial mole, detail in sections was at the resolution of the MR scanner. Nevertheless, some valuable information concerning the 3-D arrangement of the soft anatomy is apparent.

\section{ACKNOWLEDGEMENTS}

We are grateful to Ms Norah Cooper and Dr Ken Aplin of the Western Australian Museum for the loan of the specimen, and the X-ray images.

\section{REFERENCES}

Chapman, R. N. (1919). A study of the correlation of the pelvic structure and the habits of certain burrowing mammals. American Journal of Anatomy 25: 185-219.

Gambaryan, P. and Kielan-Jaworowska, Z. (1997). Sprawling versus parasagittal stance in multituberculate mammals. Acta Palaeontologica Polonica 42(1): 13-44.

Hildebrand, M. (1988). Analysis of vertebrate structure (third edition). John Wiley and Sons, Inc. New York.

Rogers, S. W. (1999). Allosaurus, crocodiles and birds: evolutionary clues from spiral computed tomography of an endocast. Anatomical Record 257(5): 162-173.

Romagnano, A., Shiroma, J. T., Heard, D. J., Johnson, R D., Sheiring, M. R., and Mladinich, C. (1996). Magnetic resonance imaging of the brain and coelomic cavity of the domestic pigeon (Columba livia 
domestica). Veterinary Radiology and Ultrasound 37(6): $431-440$.

Samii, V. F., Biller, D. S., and Koblik, P. D. (1999). Magnetic resonance imaging of the normal feline abdomen: an anatomic reference. Veterinary Radiology and Ultrasound 40(5): 486-490.

Slijper, E.J. (1946). Comparative biologic-anatomical investigations on the vertebral column and spinal musculature of mammals. Koninklijke Nederlandsche Akademie van Wetenschappen., Verhandelingen (Tweede Sectie) 42: 1-128.

Stirling, E. C. (1891). Description of a new genus and species of Marsupialia, Notoryctes typhlops. Transactions of the Royal Society of South Australia 14: 154-187.
Thomas, O. (1920). Notoryctes in north-west Australia. Annals and Magazine of Natural History (9) 6: 111-113.

Thompson, G. G., Withers, P. C. and Seymour, R. S. (2000-2001). Blind diggers in the desert. Nature Australia (Summer) 26-31.

Withers, P. C., Thompson, G. G. and Seymour, R. S. (2000). Metabolic physiology of the north-western marsupial mole, Notoryctes caurinus (Marsupialia: Notoryctidae). Australian Journal of Zoology 48: 241258.

Manuscript received 6 November 2002; accepted 9 June 2003 